\title{
Social and physical determinants of obesity in adults
}

\begin{abstract}
Obesity is one of the major health concerns in the United States. Obesity in turn leads to many other chronic health problems like diabetes, cardiovascular disease and many more. This review was done on the social and physical factors that contribute to the obesity epidemic. Social factors that were included in this review were financial stress, post traumatic stress, sleep, marriage and health literacy. Physical factors that were reviewed here were physical activity, natural environment, food environment, worksite settings, pregnancy and genetics. The data was collected from government organizations like National Institute of Health (NIH) and Centers for Disease Control and Prevention (CDC). Also, peer reviewed articles were selected based on the relevance to this review from the last five years (2011 to 2015). The result of this review was that these social and physical factors were significantly contributing to the major health problem, obesity. Overall, there has been a significant increase in the prevalence of obesity among adults in United States and this review sheds some light on the factors responsible for it.
\end{abstract}

Keywords: social factors, financial stress, marital status, weight gain, determinants, symptoms
Volume 6 Issue I - 2017

Megha Brahmbhatt

Department of Nutrition and Dietetics, Marywood University, USA

Correspondence: Megha Brahmbhatt, Department of Nutrition and Dietetics, Marywood University, USA, Email megha2259I@gmail.com

Received: March 04, 2016 | Published: January 10, 2017
Abbreviations: $\mathrm{CDC}$, centers for disease control and prevention; NIH, national institute of health; PTSD, post traumatic stress disorder; MIDUS, midlife development in the united states; BMI, body mass index

\section{Introduction}

Obesity is a complex disorder involving an excessive accumulation of body fat; it increases the risk of many health problems such as heart disease, diabetes and high blood pressure. ${ }^{1}$ Genetic and hormonal factors could also be responsible for obesity but obesity mainly occurs when the energy intake is more than energy output. According to the Centers for Disease Control and Prevention (CDC) survey of 20112012 authors, concluded that $35.1 \%$ of adults over the age of twenty were obese. Adults who are overweight including obese are $69.0 \%$ in the United States (US). ${ }^{2}$ According to the review done by National Institute of Health (NIH) the country suffers from major economic costs due to obesity; $5 \%$ to $10 \%$ of the US healthcare spending is towards the straight medical expense of obesity and overweight together. ${ }^{3}$ There could be number of other factors such as social and physical determinants that could cause overweight and obesity. Social factors could involve stress that could be financial or a stress from trauma, lack of sleep, marriage problems, and lack of education regarding health or types of food choices. Physical determinants could include a natural environment, lack of physical activity, transportation or worksite settings.

The aim of this literature review is to demonstrate reviews that have been done which shows the relationship between social and physical factors, that is significantly associated with overweight and obesity and compile it in order to create awareness among people. The country suffers from major health problems and economic costs as people might not be aware of these facts that could cause obesity or weight gain.

\section{Social determinants of obesity}

\section{Financial stress}

A study was conducted to measure the relationship of prolonged financial stress and subsequent obesity. ${ }^{4}$ In this study, the data was collected from the household income and labour dynamics Australia survey. This survey included a self-completion questionnaire and face-to-face interview that asked people about their income, health, well-being and employment. For three years in a row, this study was conducted and after completion of this Australian study the authors concluded that there was an association between prolonged financial stress and subsequent obesity. The individuals who reported to be financially stressed in two consecutive yearly surveys were more likely to be obese the following year. Other important predictors and baseline obesity were adjusted before the survey. The relationship of self-reported health, physical activity, smoking, mental health, age, marital status, ethnicity and income disappeared after controlling the baseline, but association with financial stress remained unchanged. ${ }^{4}$

This study concludes that the link between financial stress and obesity could be through both behavioural and physiological factor. Behaviourally, stress has been linked with the consumption of highly palatable foods, high fat and sweets that in turn can lead to obesity. While physiologically it shows that stress elevates the hypothalamicpituitary-adrenal activity and which is followed by metabolic abnormalities that are related to weight gain. To prevent obesity, future research can investigate behavioural factors such as dietary intake and their link between financial stress and obesity. Authors also observed that the link between obesity and financial stress was independent of mental health. So the authors concluded that financial stress could be more damaging to health compared to other forms of stress such as stress from work related matters or marital status. ${ }^{4}$ The strength of the study was that authors used a large number of nationally representative samples with a comparatively high reaction rate; the survey interview was based on in-person home interview. The weakness of the study was that it was based on self-reported height and weights; individuals who are or obese or overweight are more likely to undervalue their weight. But the research was focused on the relationship between financial stress and obesity, so underestimation of obesity is less likely to affect the results. Future investigate can examine the effect of counselling programs and financial education that will help individual with money management, saving and budgeting on a reduction in obesity and financial stress. ${ }^{4}$ 


\section{Posttraumatic stress}

A study was conducted to determine whether the women who develop posttraumatic stress disorder (PTSD) is likely to become overweight or obese subsequently, compared to the women who do not develop posttraumatic stress symptoms. ${ }^{5}$ Also, to examine whether the effects are independent of depression. This study included the subsample of the Nurses' Health Study II ages twenty-four to fortyfour years in 1989. A follow-up to 2005, in which trauma and PTSD symptoms were measured. ${ }^{5}$

The results of this study suggested that the women who had at least four or more PTSD symptoms before 1989, their body mass index increased more steeply during the follow-up years. ${ }^{5}$ While the women who developed PTSD symptoms in 1989 or later, their body mass index did not differ from their status before the onset of PTSD. The onset of four or more PTSD symptoms in 1989 or later was related to the increased risk of becoming overweight or obese among the women with normal body mass index, the result was still constant after adjusting for depression. In conclusion, this study indicates that women with PTSD were associated with faster weight gain and obesity, relative to women who did not experience any trauma or PTSD symptoms. Women with PTSD symptom onset before 1989 had higher body mass index at every follow-up study assessment, and it increased at a faster rate. This study had many positives which included a large population, body mass index before and after the onset of PTSD symptoms and extensive data on potential confounding factors. However the weakness of this study is that it was conducted only in the worst condition that could lead to misclassification of the timing of PTSD, everything was assessed retrospectively which could underestimate the lifetime prevalence. Another limitation is generalizability as the population mainly consisted of white female nurses. Findings from this study suggested that trauma will be strongly associated with increased risk of weight gain if trauma leads to the development of PTSD symptoms, although future work may also want to consider the type of trauma in this relationship more deeply. ${ }^{5}$

\section{Sleep}

A study was conducted to observe the relationship between sleep duration and obesity among adults. ${ }^{6}$ It was a meta-analysis of prospective studies; here the data was collected using websites such as PubMed and web of science. It was searched for eligible publications. After thorough research eleven published articles were included, the cut-off for sleep duration was less than or equal to five hours in some studies and six hours in other studies. Two investigators independently reviewed all the identified studies and made sure if authors met the criteria, for a short sleep duration and obesity fourteen prospective studies were selected. It was concluded that short time sleep duration was significantly associated with the risk of future obesity but out of these articles four articles reported separate results for men and women. One study's participants were just females; one study was conducted among males and the pooled measured was calculated separately for both genders; statistically significant results were obtained for both females and males. Significant association was seen in Japan and North America but not in Europe. The association of long sleep duration and risk of obesity was not significant in any of the subgroups and had no effect on weight. ${ }^{6}$

This review provided for the first time the prospective only studies meta-analysis to identify the relationship between insufficient sleep duration and risk of future obesity. But the mechanisms underlying the association between obesity and sleep were still not understood completely. ${ }^{6}$ Several potential mechanisms existed by which reduced sleep may have increased the risk of obesity. Evidence suggested that chronic sleep restriction leads to changes in measures of appetiteregulating hormones such as ghrelin and leptin, which causes the increased appetite and in turn causes increased food intake. The major strength of this study was the use of large number of participants from prospective studies, allowing for a much greater possibility of reasonable conclusions. But the weakness was that small number of studies were included in this meta-analysis so further studies are needed to confirm the findings. ${ }^{6}$

Furthermore, another study was conducted to find out the association between sleep duration and anthropometric measures among the American adults. ${ }^{7}$ In this study, the data was collected from National Health and Nutrition Examination Survey 2005 to 2010. The sleep duration for short sleepers was categorized as five hours or less and for long sleepers it was up to ten hours or more. The study participants were interviewed at home and were asked to attend a mobile examination centre where participants were asked to complete a series of questionnaire; also their blood samples were taken. The result of this study was that short sleepers had more girth and were heavier compared to long sleepers. ${ }^{7}$

These results that were gained from a big generally representative sample of adults in the United States expresses that sleep length showed opposite linear relations with waist circumference and body mass index, in groups that did not consider participants with a sleep disorder or depression. In addition, the relations did not fluctuate among three major racial or by gender. The relationship was mainly strong among adults aged twenty to thirty-nine years. Also, it was concluded that that the associations among Mexican American and African American appeared weak compared to among whites. Hispanic Americans aged eighteen to eighty-one years who slept five or less than five hours per night or eight or more than eight hours per night, raised their body mass index to a higher extent compared to those who slept six to seven hours. These results propose that further research is needed determining the associations between measures of obesity and sleep duration in Hispanic populations. The strength of the study was that it used a large population sample and the waist circumference and body mass index were measured. It had a lot of weaknesses too such as authors were not able to take into account the sleep length during weekends or non-working days. Also, the sleep duration was obtained on the basis of a self-report, so that could provide some error. This analysis did not take into account the adults without diagnosed sleep disorders or depression. ${ }^{7}$

\section{Marriage}

A study was conducted to examine if there was a relationship between marital status, obesity and body shape. ${ }^{8}$ The sample used in this study included the data from the 1995 McArthur National Survey of Midlife Development in the United States (MIDUS). The sample comprised of non-institutionalized, English-speaking adults aged twenty-five to seventy four with working telephones. Most of the sample was currently married, with men more likely than women to be married and less likely to be separated or divorced. Among the sample men got married for the first time later than the women. Women on the other hand were more likely to have two divorces and spend more time separated and to be widowed at some point in the time. ${ }^{8}$

The result of this study was that married men were heavier than divorced or separated men but did not weigh more than never-married men. However among women it was concluded that that married 
women had body weight equivalent to separated or divorced women. Never-married women had higher body mass index (BMI) and a greater likelihood of being obese than married women. Men and women who were in a live-in relationship gained less weight after age of twenty-one than did their married counterparts. Findings suggest additional factors are yet to be understood in observed associations between gender, marriage and body weight. Future research should measure the relationship between body weight and marital status among different ethnic groups. In this study, the sample did not have large number of ethnic groups to permit stratification of result through ethnicity. ${ }^{8}$

\section{Health literacy}

A study was conducted to look at the relationship between obesity and health literacy between the Pacific Islanders and Hawaiian in United States. ${ }^{9}$ The sample consisted of adults from Hawaii and Utah. A health literacy tool known as newest vital sign was used; measured demographic questions, weights and heights. The result of this study was that $45.3 \%$ of participants had at least a likelihood of low health literacy. Lower scores were linked with increased body mass index, and higher scores were linked with higher education and higher income. Women scored significantly better than men; participants' scores were not significantly different in Hawaii and Utah. ${ }^{9}$

This study had several strengths first one being the size of the sample and the second one was that participants were selected from two different states with varying economic levels. However, a convenience sample was used which limits the generalizability. Another limitation is related to data collection site and survey methods as only one measure that is newest vital sign was used, but additional health literacy measures were not used due to time limitation. Finally, the survey was done verbally in English and those who did not speak English and might find difficulties reading the nutrition facts label were obviously eliminated. Health literacy is complex, and it could be influenced by many factors. More care should be taken with elders, as lower health literacy rate was linked with advancing age and, because parents and grandparents can transmit eating habits as well as health literacy to their families. Improving their dietary choices and health literacy has the potential effect of future generations. Future research should explore other variables that could potentially influence health literacy, and such factors include health beliefs and self-efficacy and it might have a larger impact than demographic variables. ${ }^{9}$

Moreover, another study was conducted to examine if attitudes, beliefs and nutrition knowledge, were linked with obesity among lowincome African-American and Hispanic women caretaker. ${ }^{10}$ The result of the study was that Hispanics had a fair knowledge; majority of the African Americans had good knowledge of nutrition. A computed knowledge, attitudes and beliefs score had no significant relation to participants' weight status and diet quality. However, attitudes and beliefs towards healthy food strongly correlated with participants' diet consumption and weight among Hispanics. The most frequent barrier to consuming healthy foods among both the groups was the expense of healthy foods. ${ }^{10}$

It was observed that compared to Hispanics, African Americans had higher healthy eating self-efficacy, a high confidence level was seen in African American regarding making healthy food choices for their families and healthy eating. Among Hispanics language barrier could be a factor affecting their self-efficacy of healthy eating; as most of the food labels, educational materials and commercials on healthy foods are in English. African Americans had expressed that the barrier to them was the cost of healthy foods and the length of preparation. The limitation of the study was that it was a non-random, convenient sample that would limit the generalizability. Also, the diet quality did not include fats, sugar, oils and the total calories intake or expended. Lastly, the data used was from the secondary source, so authors had no control over how the data was collected or measured. Future research could focus on the requirement for nutrition educators to recognize these disparities and centre on group specific requirements as it could relate to body mass index or dietary intake. ${ }^{10}$

\section{Physical determinants of obesity}

\section{Physical activity}

A study was done to find out the association between physical activity, watching sport on television, and danger of obesity in older adults. ${ }^{11}$ The data was gathered on self-reported television time and content. Also participants' height and weight were measured, on average participants reported watching television for four to five hours a day. The result of this study was that there was no significant relationship between physical activity and watching sport. Participants who watched sports every day were at higher risk of obesity. ${ }^{11}$

The relationship between obesity and watching sports on television was particularly evident in men; this association was independent of total television viewing time as there could be number of other factors that might partly explain the effects. One of the factors could be that participants consumed more not so healthy beverages and foods while watching the television. Food advertising could also have played a role in the increased consumption of products. So this was the limitation of the study that authors were not able to take into consideration the dietary intake. Also, the data was collected based on self-report that could have created the bias in the result. In the future research should take into account the dietary intake while watching the television as it can be a major contributor to the obesity. ${ }^{11}$

Another study was done to find out the relationship between abdominal obesity, caloric intake and physical activity in American adults from 1988 to $2010 .^{12}$ It was a meta-analysis review study and authors used the national health and examination survey data. The result of the study was that the average BMI was increased by $0.37 \%$ per year in both men and women. The average waist circumference increased by $0.27 \%$ and $0.37 \%$ per year in men and women, respectively. The occurrence of abdominal obesity and obesity enlarged substantially, also the occurrence of abdominal obesity among overweight adults. Average daily caloric intake did not change significantly; younger women experienced the greatest increases in the abdominal obesity. The percentage of adults who reported no leisure-time physical activity increased from $11.4 \%$ to $43.5 \%$ in men and from $19.1 \%$ to $51.7 \%$ in women. Body mass index and waist circumference trends were linked with physical activity level but not caloric intake. The linked changes in adjusted body mass index were $1.7 \%$ higher among men and $8.3 \%$ higher among women with no leisure-time physical activity, in comparison with those with an ideal level of leisure-time physical activity. ${ }^{12}$

The limitation of this study was that the physical activity and caloric intake were self-reported, caloric intake was analyzed on a twentyfour hour recall. So the caloric intake could have been underestimated, the physical activity levels could have been overestimated but this did not explain the higher rates of no leisure-time physical activity in the study. Future research should promote this message about nutrition and physical activity. Make physical activity as an integral part and 
a major focus stressing the roles of health care providers, employers, and schools in promoting health. ${ }^{12}$

\section{Natural environment}

A study was conducted to look at the relationship between obesity and neighbourhood environmental characteristics, also associated behaviours among adult New Zealanders. ${ }^{13}$ This study used individual-level data from the 2006-2007 New Zealand health survey on diet, obesity, physical activity and socio-economic variables correlated to geographic information from other sources related to environmental factors, based on the respondent's residential address. The result of this study was positive, and it was concluded that that decreased access to neighbourhood green space and neighbourhood deprivation were both significantly linked with the greater than before odds of obesity and overweight. The result also suggests that intensity of physical activity per week was linked with rural and urban status; less activity among those living in the areas with higher levels of active travel to work and higher activity in more rural areas. Also, there was an important tendency for low levels of walking linked with neighbourhood scarcity. ${ }^{13}$

The study had few limitations, first one being that the data used for health and behaviour were cross-sectional, and it limits the ability to conclude the causality. Also, the authors did not take into account the length of residence in the neighbourhood. Lastly, all the weight related behaviours were self-reported. Future research should improve upon these limitations; also individuals may meet a friend in their neighbourhood for a walk or exercise at a park. As such proportion of time spent in each area would progress the understanding of the environmental determinants of this global epidemic, obesity. ${ }^{13}$

Furthermore, another study was conducted to examine the relationship between obesity and urban environment in south-east Asia. ${ }^{14}$ This study was a meta-analysis review, which was done in order to provide a review of studies that showed the positive association between obesity and urban living environment. The authors claimed it to be the first study to research this relationship in south-east Asia. There were twenty-eight studies that included adults in this review; studies were from Thailand, Vietnam, Malaysia, Myanmar, Philippines, Indonesia and some other. Out of these six nations met the criteria set for the study. The results of this study demonstrated a positive relationship between obesity and urban living in south-east Asian countries. It was consistent with all age groups including both genders. $^{14}$

There were certain limitations to this study, first one being that this study compared the outcomes in rural and urban settings. ${ }^{14}$ This may have missed the underlying factors that could have been potentially linked to obesity, such as the duration of stay in the area, economic diversity and commute to the workplace during the year. Also, this review might not have included all the relevant studies that have been conducted to look at the association between obesity and urban living. The major strength of this study was that it collected all the information from relevant sources like PubMed and Global Health. Future research should explore more into the underlying factors that could be important to confirm the relationship between obesity and urban living. Such factors could be the length of stay in the area, economic diversity and also the transport to work during the year. ${ }^{14}$

\section{Food environment}

A study was conducted to examine if there was a positive relationship between exposure to take away food outlets, body weight and take away food consumption. ${ }^{15}$ Here the commuting route, home and workplace environments were also taken into account and was conducted for working adults in the United Kingdom (UK). The authors of this study claimed that this was the first study to offer the complete evaluation of exposure to these outlets, measured body mass index and consumption of energy-dense takeaway foods. ${ }^{15}$

The result of this study was that there was a positive link between takeaway food consumption and body mass index in all exposure domains of work, commuting environments and home and the high odds of obesity. It estimates that participants who were exposed the most consumed $15 \%$ higher than those who were least exposed, which would be additional $5.7 \mathrm{~g}$ per day for takeaway food. Among the three domains, the greatest effect was for environmental relationship that concluded the takeaway food consumption at work. In context, to odds of obesity and body mass index similar relationship was seen; with the maximum takeaway food consumption at home compared to at work. There were a few limitations to this study; the distance that could be covered by an adult in fifteen minutes was defined as neighbourhood. This definition of neighbourhood could vary between work and home and also from person to person. Also, authors did not take into consideration the types of takeaway food outlets available at the workplace so the food could have been bought from there such as office canteen. It was also not mentioned how long the participants were exposed to this environment, both at work and home. Future research should focus on these limitations. Also, it should explore the effects of exposure to the food environment on unemployed people and people with low socioeconomic status, as they might be more restricted to these built-up neighbourhoods. ${ }^{15}$

Moreover, another study was conducted to examine the relationship between body mass index and neighbourhood density restaurants in rural Chinese adults. ${ }^{16}$ It was a longitudinal study, and the data was collected from the China Health and Nutrition Survey. The result of this study was positive; neighbourhood restaurants density was significantly associated with the body mass index in rural China. The increment of one indoor restaurant in the neighbourhood resulted into 0.005 increase in the body mass index among women whereas 0.01 for men. $85 \%$ of the participants who responded were married. The association was concluded that negative between body mass index and neighbourhood fast food restaurants in rural women. Individual level variables were also taken into consideration. It was seen that married adults had lower body mass index compared to unmarried adults and also participants who were physically active had lower body mass index compared to those who were not. ${ }^{16}$

Compared to eating at home people tend to eat more energy densefoods, large portion sizes and also increased alcohol consumption when they eat out, same was the case in this study. The major strength of this study was the use of large scale longitudinal data. The limitation was that this study did not include any of the restaurants outside or nearby the neighbourhood that may have influenced the results. The clear relationship between body mass index and restaurant density was not defined. Also, some underlying factors were not taken into consideration such as frequency of eating out or the location of home that also might have influenced the result. Future research should take this into account. Also, it should examine the effect of these neighbourhood restaurants on eating behaviours and individual dietary intake. ${ }^{16}$

Furthermore, another study conducted to determine the relationship between urban food environments and obesity in adults. ${ }^{17}$ Data for this study was collected through telephonic interviews in 
which the participants were asked about their body weight and also individual characteristics were collected. The result of this study was that the greater access to convenience stores and fast food restaurants was associated with greater risk of being obese. The majority of the participants were females and the prevalence of obesity among the sample was $26.5 \%$, African-Americans, older adults and females were affected more by obesity. It was also observed that participants with low incomes and less education had higher rates as well. The study had some limitations, first one being that the heights and weights were self-reported so the body mass index calculated could have been underestimated. Another limitation here was that some participants did not mention body mass index or other individual variables and still they were included in the study that could have formed a bias as the missing body mass index showed no association with obesity prevalence. Lastly, there could be number of other factors that showed the difference in obesity odds that were unobserved. It could be neighbourhood poor walkability or high crime rates that could have affected the physical activity levels of participants and in turn the weight status. ${ }^{17}$

This was a cross-sectional study, so future research could conduct experimental and longitudinal studies to find out the associations between food environment and obesity. Also, determine if the environment is modified it helps to prevent or reduce this obesity epidemic or not. ${ }^{17}$

\section{Worksite settings}

A study was conducted to look at the association between work site environment and individual impact on sugar-sweetened beverage (SSB) consumption among the employees who were obese. ${ }^{18}$ Most participants in this study were white and had some college education or degree, here the weight and height status of the participants were measured. Also, the questionnaire in relation to beverage intake was completed. The result of this study was positive, it was observed that participants consumed $17 \mathrm{fl} \mathrm{oz}$ of SSB that was higher than the recommended discretionary energy intake, and the mean water consumption was $27 \mathrm{fl} \mathrm{oz}$. Lower SSB consumption was concluded that among participants with high household income and higher education level. Also, baseline body weight was associated with a greater number of vending machines and worksite water coolers, which in turn was linked with higher SSB consumption. ${ }^{18}$

The strength of this study was that it included detailed information on the beverage consumption. ${ }^{18}$ There were nineteen types of beverages listed on the questionnaire and data was collected using a validated tool and also had a large sample size. But it had few limitations as well, first being that the participants for this study were obese or overweight adults so beverage patterns were not compared with the general population or the people with normal weight status. Also, there was no examination of the role of caffeine among the beverages; habitual beverage consumption was a self-reported data that could have underestimated the results. Finally, the participants in this study mainly consisted of white women that limit the generalizability. Future research should examine whether increasing water consumption and reducing SSB consumption at the work sites is a useful intervention method for weight management. It should also see whether environmental or individual intervention factors improve the results. ${ }^{18}$

Moreover, another study was conducted to look at the association between the weight-related behaviours, body mass index and work hours per week among the metro transit workers. ${ }^{19}$ A survey was conducted in which participants were asked to report about their food choices, leisure time physical activity, and how many hours a week they worked. Also, they were asked to mention their perception of a work environment in regards to eating healthy.

The result of this study was that participants who worked the greatest number of hours which was fifty hours or more per week had poorer dietary habits and highest body mass index. ${ }^{19}$ The results differed by gender as well; more hours per week were related to higher body mass index in women compared to men. Working more hours for men was linked with more access to vending machines for snacks, cold drinks and cold food and on the other hand for women it was linked to more consumption of vegetables and fruits. For participants working forty hours or more per week, there was higher consumption of cold drinks, cold foods and snacks from a vending machine among women compared to men. Also, the perception of eating healthy was not linked with work hours in either women or men. This study had some strengths including the large sample size that included both genders; male and female. Several factors were taken into consideration to gain proper knowledge about the weight status which included participants eating behaviour, dietary intake and how often do they engage in leisure time physical activity. But the study had few limitations as well, the first one being that the measures of physical activity and dietary intake were self-reported so it could have been inaccurate and in turn might have underestimated the result. Another was that the sample size in this study had very low numbers of participating women, which was only $6 \%$ of working women were in the highest working hour category of fifty hours. This might have limited the gender stratified associations. This study has taken into account only one work-related factor that was the number of hours worked. Future research should focus on other factors such as job stress, job flexibility and work shifts that could have been linked with health related behaviour. Interventions to provide more healthy food choices at the work sites could also help. This study clearly shows how eating unhealthy foods resulted in increased body mass index among the transit workers. ${ }^{19}$

\section{Pregnancy}

Pregnancy could also be one of the factors contributing to the epidemic obesity. One study was conducted to determine how prepregnancy weight, gestational weight gain and postpartum weight changes influences body mass index and maternal weight seven years after delivering a baby. ${ }^{20}$ Here women bearing one child and no births during the follow-up were selected. The results showed a positive outcome in which women had postpartum weight retention at six months and weight gain from six to eighteen months postpartum. So the average increase in the weight was $0.5 \mathrm{~kg}$ during seven years. It was also concluded that that breastfeeding had the inverse relationship with weight status. Especially the effect was greatest for waist circumference, so this shows that breastfeeding may have a beneficial effect. $^{20}$

These results demonstrated that the first eighteen months post pregnancy is crucial to prevent obesity related to pregnancy. ${ }^{20}$ This study examined the weight status during different periods and also the indirect and direct mediating effects which is the strength of the study. Also, the study population was large which allowed the authors to restrict the study population to women who did not give birth during their follow-up. But the study had few limitations, as well as this, was an observational study perplexity cannot be ruled out. The data collected was based on a self-report that could have affected the results; it was observed that an average of $0.66 \mathrm{~kg}$ pre-pregnancy 
weight was underreported throughout the entire body mass index scale. But it was assumed that this underreporting might have been present for postpartum weights as well and therefore did not affect the estimates. There could also have been a possibility that those women who gained a lot of weight seven years after delivering the baby were less likely to take part in the follow-up study. But authors had no data examining this. It also limits the generalizability as the participants mainly consisted of white women. Future research should examine the effects of breastfeeding on weight status in more detail, as this study shows some beneficial effects of it on weight status. Also, additional studies should be conducted in which the weights should be measured rather than self- report that would help confirm the findings of this study. Finally, future research should also conduct the same type of study including the participants from different ethnicity so that the result could be generalizable. Here authors just assumed that the findings of this study could be applied to the European population with a western lifestyle. ${ }^{20}$

\section{Genetics}

A study was conducted in Canada to review the relationship between behavioural and genetic findings in relation to adiposity, body weight, obesity and adipose tissue distribution. ${ }^{21}$ This was an observational study; first authors looked at the studies related to genetics of obesity; the result was that there was significance for various phenotypes of obesity with familial resemblance. Authors compared within versus between family variations; it showed that there was a higher variation, between families than within families in relation to body composition, body fatness and also for energy intake and expenditure. The overall changes or variations were more in the subjects sharing both familial environment and genes compared to the ones sharing only familial environment. ${ }^{21}$

Further studies were conducted in order to examine how familial resemblance could affect the genetic differences and also to examine the heritability of underlying phenotypes. This study showed that there was a significant effect of familial resemblance on genetics. This lead to heritability estimates that ranged from $56 \%$ to abdominal visceral fat and $42 \%$ for subcutaneous abdominal fat. ${ }^{21}$ This study was a review of previous studies that has been done so far on genetics and how it could affect the epidemic obesity. The limitation is that there was no mention in detail about how authors conducted these studies as it was an observational study. Through this review authors also concluded that out that the heredity could also play a role in exercise intensity and alcohol consumption, it had a major influence on adiposity. Future research should look at this relationship in more detail as genetics could play a significant role in weight status of an individual. ${ }^{21}$

\section{Discussion}

This review highlighted the significant factors that contribute to the public health crisis of obesity in United States. The average waist circumference, prevalence of abdominal obesity, prevalence of obesity and average body mass index (BMI), increased substantially over the past three decades in both men and women. The obesity epidemic is likely to grow consistently and soon become the leading health issue if no effective approaches are implemented to control or stop it. Native Americans, some minority and low socio-economic status groups such as Africans and Hispanics and also Pacific Islanders are affected by obesity. This review mainly demonstrated some of the physical and social factors that could be responsible for obesity. A superior understanding of the factors that have contributed to the differences across groups and the underlying factors that initiated the increase in obesity incidence in the United States over the past few decades is crucial in combating this rising community health calamity.

This review certainly has a limitation. There could be number of other social and physical factors which are not included here. It could be factors like the level of partying or how much and how often does a person consumes alcohol. Also the effects of when a person moves to another country or the environment different from where they grew up and many more. Future research should focus on these other factors that are also contributing towards the health crisis, obesity. Without developing efficient plans to alter the current obesogenic surroundings in the United States, it is possible that the obesity plague will grow even more. Government agencies, individual persons, industry and public health professionals all need to play a dynamic role in the rising national hard work to battle the obesity epidemic.

\section{Conclusion}

The goal of Healthy People $2020^{22}$ for the topic of nutrition and weight status is to promote health and to reduce the risk of chronic diseases through exercise and healthy diets and maintenance of healthy body weight. Continued advances in obesity research, detection and treatment have resulted in an increase in both incidence and death rates caused due to obesity. The aim of this paper was to provide a review of social and physical factors that are associated with obesity, most of the agenda focuses on the causes that could lead to obesity. Social factors like stress, health literacy, sleep and marriage are discussed in this review as these are the significant factors that could cause obesity. Also, physical factors like genetics, physical activity, food environment, worksite settings, natural environment and pregnancy are discussed and how these play a major role in contributing towards obesity. This review will hopefully help in the prevention of weight gain or possibly the ability to control the determinants of obesity. Further research is required to continue to provide a growing body of evidence on the impact of social and physical factors on obesity.

\section{Acknowledgements}

None.

\section{Conflict of interest}

The author declares no conflict of interest.

\section{References}

1. Mayoclinic staff. Obesity, Mayoclinic. USA; 2013.

2. National Center for Health Statistics. Obesity and overweight. Centers for Disease Control and Prevention, USA; 2015.

3. Adam GT, W David F, G Henry A Direct medical cost of overweight and obesity in the United States: a quantitative systematic review. Obes Rev. 2011;12(1):50-61.

4. Siahpush M, Huang TT, Sikora A, et al. Prolonged financial stress predicts subsequent obesity: Results from a prospective study of an Australian national sample. Obesity a Research Journal. 2013;22(2):616-621.

5. Kubzansky L, Bordelois P, Jun H, et al. The weight of traumatic stress: A prospective study of posttraumatic stress disorder symptoms and weight status in women. Journal of American Medical Association Psychiatry. 2014;71(1):44-51.

6. Wu L, Zhai L, Zhang D. Sleep duration and obesity among adults: A metaanalysis of prospective studies. Sleep Medicine. 2014;15(12):1456-1462. 
7. Ford ES, Li C, Wheaton AG, et al. Sleep duration and body mass index and waist circumference among US adults. Obesity a Research Journal. 2013;22(2):598-607.

8. Jeffery S, Karla H. Marital status, marital history, body weight, and obesity. Marriage \& Family Review. 2011;47(7):474-504.

9. Lassetter JH, Clark L, Morgan SE, et al. Health literacy and obesity among native Hawaiian and Pacific Islanders in the United States. Public Health Nursing. 2015;32(1):15-23.

10. Irene A, Lauren H. Are nutrition knowledge, attitude and beliefs associated with obesity among low Hispanic and African American women caretakers? Journal of Obesity. 2013;2013:8.

11. Mark H, Richard W, Emmanuel S. Watching sport on television, physical activity and risk of obesity in older adults. BioMed Center of Public Health. 2014;14:10.

12. Uri L, Ajitha M, Parvathi M, et al. Obesity, abdominal obesity, physical activity and caloric intake in US Adults: 1988 to 2010. The American Journal of Medicine. 2014;127(8):717-727.

13. Amber L, Graham B, Peter D, et al. Associations between neighbourhood environmental characteristics and obesity and related behaviours among adult New Zealanders. BioMed Center of Public Health. 2014;14:553.

14. Angkurawaranon C, Jiraporncharoen W, Chenthanakij B, et al. Urban environments and obesity in Southeast Asia: A systematic review, metaanalysis and meta-regression. PloS ONE. 2014;9(11):e113547.

15. Burgoine T, Forouhi N, Griffin S, et al. Associations between exposure to takeaway food outlets, takeaway food consumption, and body weight in Cambridgeshire, UK: Population based, cross sectional study. British Medical Journal. 2014:348.
16. Du W, Su C, Wang $\mathrm{H}$, et al. Is density of neighbourhood restaurants associated with BMI in rural Chinese adults? A longitudinal study from the China Health and Nutrition Survey. British Medical Journal. 2014;4:e004528.

17. Bodor N, Rice J, Farley T, et al. The association between obesity and urban food environments. Journal of Urban Health. 2010;87(5):771781 .

18. Davy B, Almeida F, Wall S, et al. Impact of individual and worksite environmental factors on water and sugar-sweetened beverage consumption among overweight employees. Preventing Chronic Disease. 2014;11:130207.

19. Escoto K, French S, Harnack L, et al. Work hours, weight status, and weight-related behaviours: A study of metro transit workers. International Journal of Behavioural Nutrition and Physical Activity. 2010;7:91.

20. Kirkegaard H, Stovring H, Rasmussen K, et al. How do pregnancyrelated weight changes and breastfeeding relate to maternal weight and BMI-adjusted waist circumference $7 y$ after delivery? Results from a path analysis. The American Journal of clinical Nutrition. 2014;99(2):312319

21. Chaput J, Perusse L, Despres J, et al. Findings from the Quebec family study on the etiology of obesity: Genetics and environmental highlights. Curr Obes Rep. 2014;3:54-66.

22. Healthy People. Nutrition and weight status. 2015. 